Brit. Heart J., 1964, 26, 452.

\title{
THE HÆMODYNAMIC IMPLICATIONS OF THE BISFERIENS PULSE
}

\author{
BY \\ H. IKRAM*, P. G. F. NIXON*, AND J. A. FOX \\ From the Department of Thoracic Surgery in the General Infirmary, Leeds, \\ and the Department of Civil Engineering, the University of Leeds \\ Received August 27, 1963
}

The indirect arterial pulse tracing from a healthy young person normally shows two systolic waves, the percussion wave and the tidal wave (Fig. 1). In hypertension and old age the percussion wave is usually small or absent (Fig. 2). In health, anæmia, and hyperthyroidism (Hancock and Abelmann, 1957), and in patent ductus arteriosus (Fig. 3), the two peaks may be prominent enough to give the pulse a bisferiens character. In aortic valve disease the pulse has a water-hammer quality when the percussion wave is large and sharply peaked, and an anacrotic quality when it is absent or small. The bisferiens pulse is known to occur in aortic valve disease (Bramwell, 1937) (Fig. 4), but there is little agreement about either the nature of the responsible valve lesion or the genesis of the pulse.

The purpose of this communication is to describe the nature of the aortic valve lesion in a series of patients with a bisferiens pulse, and to consider the factors that may be responsible for the character of the pulse.

\section{SubjeCtS AND Methods}

The patients studied were candidates for the surgical treatment of their disabling aortic valvular disease, and in each the clinical impression of a bisferiens carotid pulse was confirmed by an indirect carotid arterial tracing. Cardiac catheterization was carried out when they were quietly resting and in a basal or close to basal state. A $90 \mathrm{~cm}$. red Kifa catheter was introduced percutaneously into the femoral artery and passed to the ascending aorta. The left ventricle was entered with a $90 \mathrm{~cm}$. PE.50 catheter introduced through a Ross transseptal needle (Ross, 1959). Pressure pulses were measured with Statham P23G transducers, and recorded synchronously with the indirect carotid arterial tracing. The degree of aortic regurgitation was assessed by the Braunwald technique (Braunwald and Morrow, 1958): blue dye was injected into the aorta at various levels, and the lowest point in the descending aorta from which the blue dye regurgitated back to the ascending aorta and perfused the right ear was determined with an ear-oximeter. The regurgitation was considered to be moderately severe if the lowest point for the detection of regurgitation was at the level of the lower part of the pulmonary arc, severe if it was at the level of the left dome of the diaphragm, and gross if it lay below the diaphragm. Tachycardia was not present in any case.

Indirect Carotid Arterial Tracing. The lightly-sprung probe of a displacement-measuring instrument (Schneider and Klunhaar, 1961; Nixon, Hepburn and Ikram, 1964) was used to record the movement of the skin overlying a point of maximum pulsation of the right carotid artery in the upper part of the neck, where the artery is resting against unyielding tissues. The physician feels this movement when he palpates the pulse with his fingers. This displacement-measuring apparatus amplifies and records uniformly over an adequate frequency range, i.e. without the signal being distorted by resonance artefacts, or the velocity or the acceleration components of the movement, within the range 0.02-20 c.p.s.

* Present address: Charing Cross Hospital, London W.C. 2. 

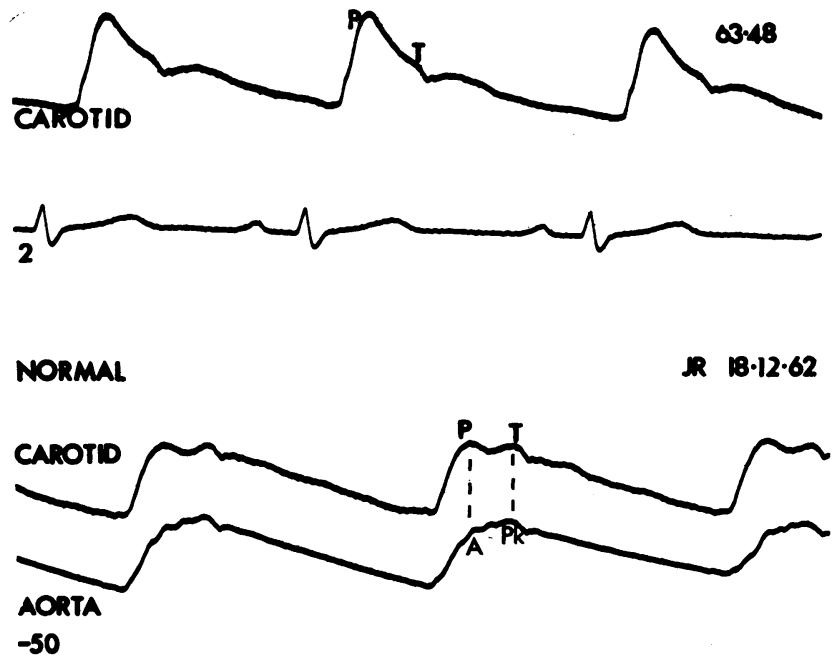

$-0.450 x-1$

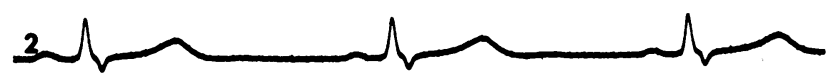

Fig. 1.-Carotid arterial displacement curves from two healthy youths. The lower is recorded simultaneously with the aortic pressure pulse. $\mathbf{P}$ and $\mathrm{T}=$ percussion wave and tidal wave. $\mathrm{A}$ and $\mathbf{P k}=$ anacrotic wave and peak. $2=$ lead II electrocardiogram.
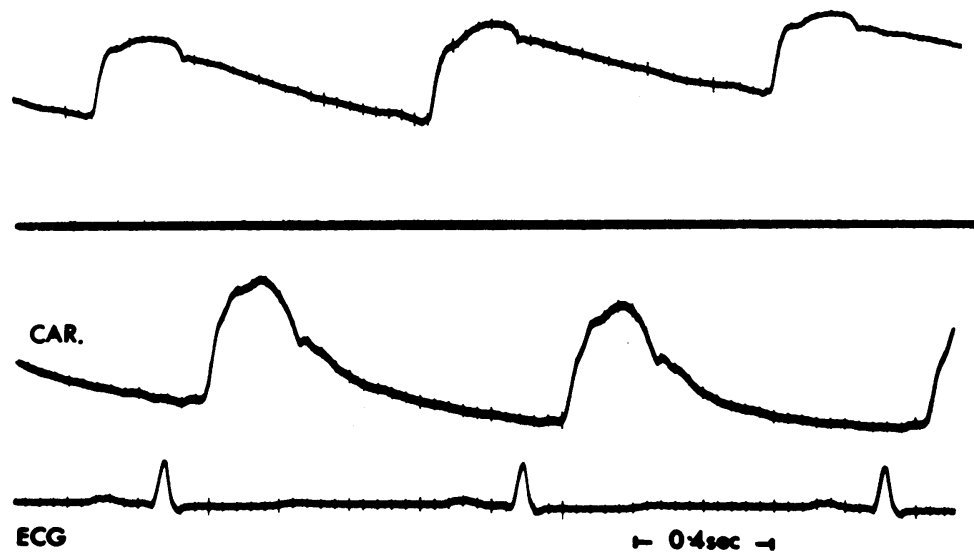

Fig. 2.-Carotid arterial displacement curves from an aged person (upper tracing), and a hypertensive patient (lower tracing).
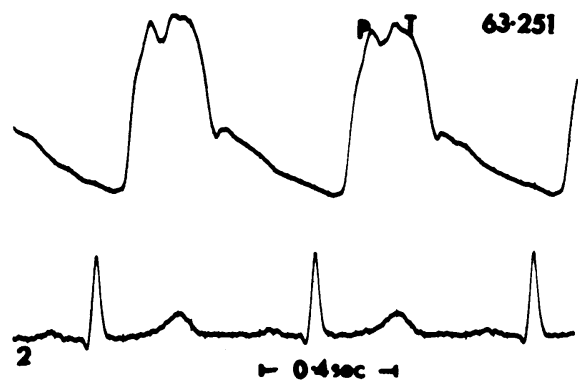

Fig. 3.-Carotid arterial displacement curve from a patient with patent ductus arteriosus. Abbreviations as in Fig. 1. 


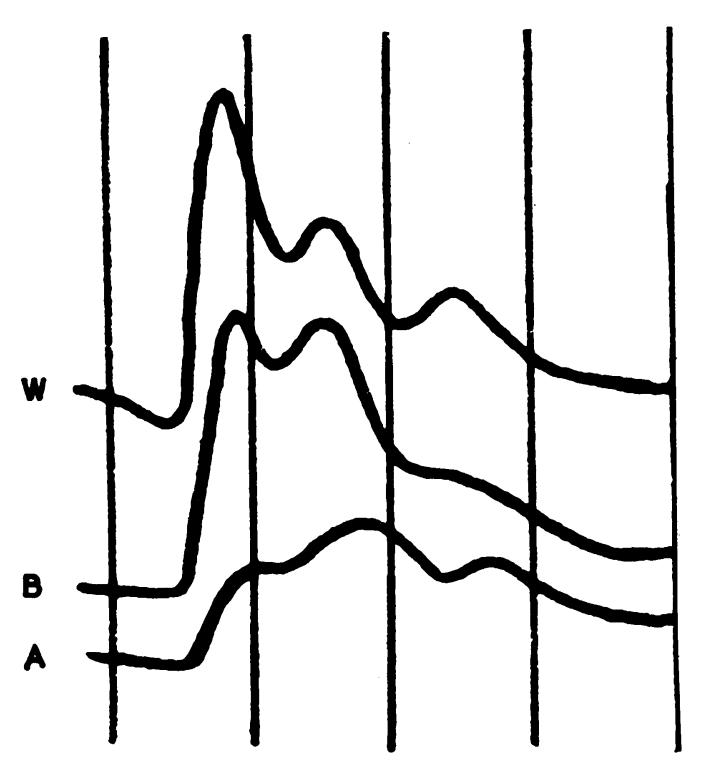

FIG. 4.-Bramwell's illustration (1937) of the waterhammer (W), bisferiens (B), and anacrotic (A) types of arterial pulse in aortic valve disease.
The patient was included in the study if the carotid tracing resembled Bramwell's illustration (Fig. 4) but not if it was anacrotic, water-hammer, or intermediate in pattern.

Terminology. The terms percussion wave and tidal wave describe the first and second systolic peaks of carotid arterial displacement curve; the corresponding points on the aortic pressure pulse are referred to as the anacrotic wave and the peak (Fig. 5).

\section{RESULTS}

Five patients had moderately severe aortic regurgitation. Their ages lay between 21 and 44 years. Their blood pressures (sphygmomanometer) were $150 / 50,210 / 70,140 / 50,140 / 60$, and $180 / 40 \mathrm{~mm}$. $\mathrm{Hg}$. In three, the peak left ventricular pressure exceeded the peak aortic pressure by 35,10 , and $15 \mathrm{~mm}$. $\mathrm{Hg}$ respectively, and in 2 it did not exceed the peak aortic pressure. In 2, mitral regurgitation was present in addition to the aortic lesion. Two patients, aged 29 and 33 years, had severe aortic regurgitation. Their blood pressures were 170/30 and $110 / 50 \mathrm{~mm} . \mathrm{Hg}$ : in one the peak left ventricular pressure was not higher than the aortic, and in the other it exceeded it by $50 \mathrm{~mm} . \mathrm{Hg}$. Three patients, aged 25 to 33 years, had gross aortic regurgitation. Their blood pressures were $110 / 30,140 / 15$, and $200 / 40 \mathrm{~mm}$. Hg. In none was the peak of left ventricular pressure higher than the peak of aortic pressure. In one a ventricular septal defect was present.

These results indicate that a bisferiens carotid pulse may occur in association with moderately

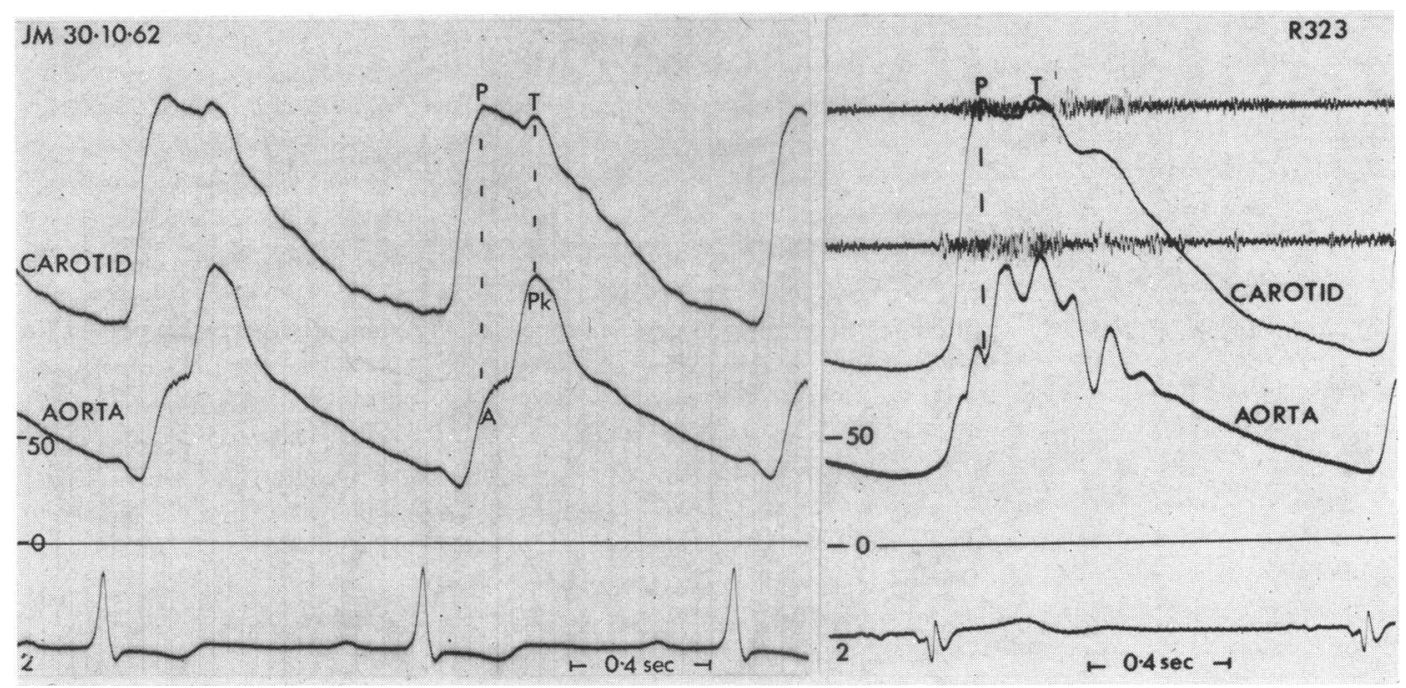

FIG. 5.-The bisferiens carotid arterial displacement curve in two patients with aortic regurgitation. Abbreviations as in Fig. 1. 
severe, severe, or gross aortic regurgitation. The absence of an aortic peak systolic pressure gradient in the gross cases suggests that the regurgitation was "pure", i.e. the valve lesion was free from an element of stenosis. This impression was confirmed at operation in one case where the aortic incompetence was caused by the prolapse of a valve cusp into a ventricular septal defect.

In 4 of the 7 patients with lesser degrees of regurgitation the presence of aortic peak systolic pressure gradients measuring from 10 to $50 \mathrm{~mm}$. Hg suggests the possibility of a degree of stenosis. Flow gradients, if they existed, would be more likely to occur in the cases of gross regurgitation.

The bisferiens pulse has not been encountered by the authors in pure aortic stenosis, nor in aortic stenosis with slight regurgitation.

\section{Discussion}

The Nature of the Aortic Valve Lesion Associated with a Bisferiens Pulse. The bisferiens pulse has been observed in cases of pure aortic regurgitation by Steell (1894), Clarke (1894), Broadbent and Broadbent (1900), Lewis (1906), Hancock and Abelmann (1957), and H. Hartman (personal communication, 1963); and Mackenzie (1908, Fig. 147) and Robinson (1963, Fig. 3a) have published typical bisferiens tracings from patients with aortic incompetence. Fleming (1957) has recorded the finding of a bisferiens aortic pressure pulse in cases where there was evidence of "significant" aortic regurgitation after aortic valvotomy. Broadbent and Broadbent (1900), Mackenzie (1908), Wood (1956), and Robinson (1963) considered that an element of stenosis had to accompany the incompetence before the pulse could assume a bisferiens character, but little pathological or physiological evidence is presented in support of their claim, and one wonders if it was the presence of the bisferiens pulse itself, and the absence of a water-hammer pulse, which led these authors to their conclusion. Apart from the lack of physiological or pathological correlations, there are two other considerations that make it difficult to draw conclusions from the literature on the bisferiens pulse. First, a variety of recording techniques have been applied to different arteries: it may be impossible, for example, to compare the results of a study of the aortic pressure pulse with conclusions drawn from the radial sphygmograph, or to decide how reliably an author could distinguish by palpation the difference between an anacrotic and a bisferiens pulse. Second, it may not be easy to understand the criteria used by an author for his definition of the bisferiens pulse: for example Mackenzie (1908, Fig. 147, 148) and Robinson (1963, Fig. 3a) have each published pulse tracings from cases of aortic regurgitation which are, in our opinion, more floridly "bisferiens" than the tracings presented as typical examples of the condition; and Feil and Gilder (1921, Fig. 6) have published an example of the anacrotic pulse that other clinicians might well have called bisferiens.

In aortic valve disease intermediate pulse forms that do not resemble closely any of the patterns shown in Fig. 4 are commonly encountered, but they have not been included in this study which shows that the bisferiens pulse can and does occur in "pure" severe aortic regurgitation as well as in cases where there is probably an element of stenosis.

The Nature of the Bisferiens Pulse. Simultaneous records of the carotid displacement pulse and the aortic pressure pulse show that the carotid percussion wave occurs at the time of the anacrotic wave of the aortic pressure pulse. The tidal wave coincides with the peak of aortic pressure (Fig. 1, 5), and is not a reflected wave. This observation agrees with Robinson (1963).

The feature that appears to be characteristic of the bisferiens carotid pulse is the early systolic appearance of a rapidly rising percussion wave of displacement that is much more prominent than the synchronous anacrotic aortic pressure wave (Fig. 5); and it is reasonable to assume that this indicates the achievement of a sudden relatively large increase in the volume of blood contained in the artery for a relatively small increase in the aortic pressure. The percussion wave may be regarded as a transient pulsation imposed under certain circumstances on the basic arterial wave form, and the trough following may be attributed to its subsidence. The disproportionate enlargement of the percussion displacement wave reaches an extreme in the water-hammer pulse (Fig. 6). Bisferiens and water-hammer patterns of pulse, albeit on a small scale, are common findings in 


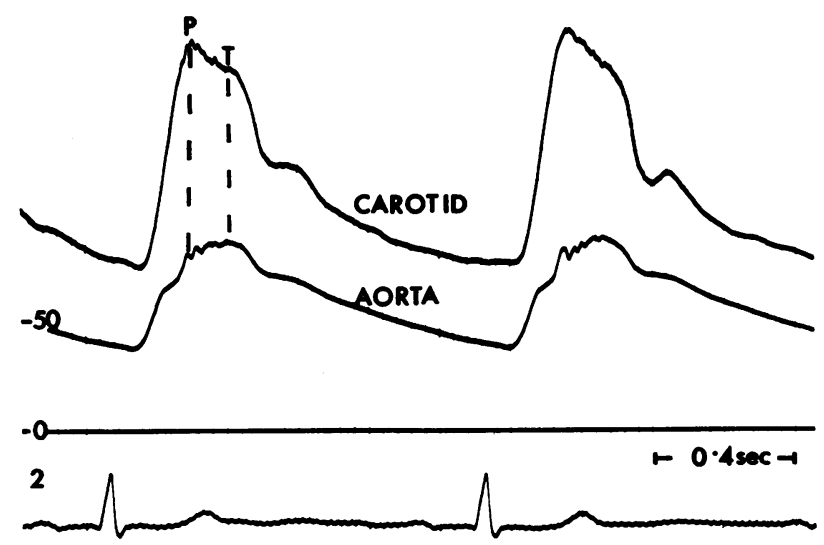

FIG. 6.-The water-hammer carotid arterial displacement curve in aortic regurgitation, with the simultaneously recorded aortic pressure pulse. Abbreviations as in Fig. 1.

young healthy people, and the limited evidence that we have (Fig. 1) suggests that the disproportion is also present.

In hypertension and old age the absence or the poverty of the percussion wave (Fig. 2) indicates that the disproportion is slight, and it is reasonable to conclude that a relatively small increase in aortic pressure cannot produce a large increase in the carotid arterial volume. In aortic stenosis the valvular lesion tends to prevent the sudden early systolic filling of the arteries, and there is usually little disproportion between the carotid displacement pulse and the aortic pressure pulse (Fig. 7). In 1937 Bramwell observed that aortic stenosis affected the pulse contour in a manner similar to hypertension.

Theoretically the important factors in the relation between the carotid displacement pulse and the aortic pressure pulse are the condition of the arterial wall, and the magnitude and the rate of change in blood volume within the vessel.

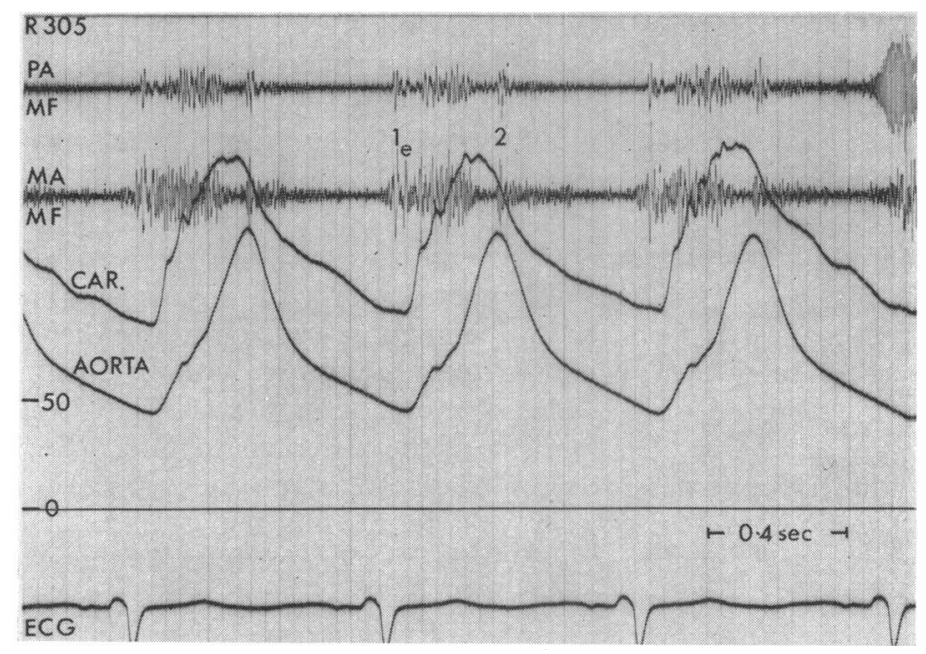

Fig. 7.-The anacrotic carotid arterial displacement curve (CAR) in aortic obstruction, with the simultaneously recorded aortic pressure pulse, the pulmonary (PA/MF) area and mitral area (MA/MF) phonocardiograms, and the electrocardiogram. I,e 2 =first heart sound, ejection sound, and second sound. 
The Arterial Wall. In 1937 Bramwell drew attention to the fact that when an artery is first put on stretch the readily extensible fibres of the wall govern its behaviour, and a considerable increase in the volume of the artery may be produced for a relatively small increase in pressure; as the pressure rises the more inextensible fibrous tissue of the wall becomes tensed, and resists further stretching. It follows that conditions that increase the diastolic emptying of the arteries must predispose them to ready distensibility in early systole, and tend to produce a water-hammer or a bisferiens type of pulse: examples of such conditions are aortic incompetence, patent ductus arteriosus (Fig. 3, 5), and states of vasodilatation. The increase in the radial percussion wave with elevation of the arm may be attributed to enhanced diastolic emptying of the artery. It is interesting to note that Hope (1839) regarded the pulse of aortic regurgitation as the "pulse of unfilled arteries". Compression of the artery (Steell, 1894; Broadbent, 1899), tensing the artery (Morison, 1894), hypertension, and the stiffening of old age (Bramwell, 1937) tend to have an opposite effect.

Perhaps the preservation of a large and rapid initial rise in the carotid percussion wave in young children with severe aortic stenosis (Robinson, 1963) may be attributed to a readily distensible condition of the young arterial wall.

The Magnitude and the Rate of Change of the Blood Volume within the Artery. Aortic regurgitation, patent ductus arteriosus, and the other conditions that enlarge the stroke output and the pulse pressure probably enhance the early-systolic filling of the arteries and tend to produce a bisferiens or water-hammer type of percussion wave if the vessel wall is in a readily distensible condition. Bramwell (1937) remarked that any condition causing a high pulse pressure and a low diastolic pressure tended to produce a pulse resembling that of aortic incompetence. As will be seen later, there is evidence to suggest that a large stroke output may be responsible for the bisferiens or collapsing type of arterial pressure pulse.

Aortic stenosis impedes the early systolic filling of the arteries and must oppose the production of the bisferiens and water-hammer types of percussion wave.

The Anacrotic Aortic Pressure Wave. The work of Spencer and Greiss (1962) indicates that the anacrotic wave of the aortic pressure pulse corresponds with the period of acceleration of the blood flowing through the aortic valve, when the left ventricular pressure is higher than the aortic. Later in the ejection phase the blood flow decelerates and the aortic pressure is higher than the left ventricular pressure. The maximum alteration in the rate of change of momentum of the blood flow occurs at the time of the peak of the anacrotic pressure wave, and it is to be expected that the magnitude of the peak will be proportional to the alteration in the rate of change of momentum. During the spread of a pressure wave towards the periphery of the arterial system the "front" and the peak increase in amplitude (McDonald and Taylor, 1959), and consequently the anacrotic waves tend to become more prominent.

Enlargement of the stroke volume appears to be a common finding in patients with a bisferiens pulse, and it is reasonable to suppose that it predisposes to a high initial ejection velocity, to a large alteration in the rate of change of momentum of the ejected blood, and, consequently, to a prominent anacrotic wave. The amplitude of the corresponding percussion wave of the carotid displacement pulse is modified by the condition of distensibility of the arterial wall.

The depth and shape of the trough between the anacrotic wave and the peak of the aortic pressure pulse, or between the percussion and the tidal waves of the carotid arterial displacement pulse, depend upon the magnitude and slope of the two positive waves, and there is no need to postulate a "Venturi" mechanism (Fleming, 1957) to explain its presence. The application of the Venturi principle to pulsatile blood flow in elastic vessels is not yet established, and records of the brachial and femoral arterial displacement pulses from a case of coarctation of the aorta suggest that a constriction may tend to obliterate the percussion peak and trough (Fig. 9).

The Clinical Implications. There is a conflict of opinion about the value of pulse tracings in aortic valve disease, and some of the reasons for the disagreement have been discussed. It appears that youth, a low diastolic blood pressure, a large stroke volume, and aortic regurgitation tend to produce a bisferiens or collapsing type of carotid percussion wave, while advancing age, a rise in 


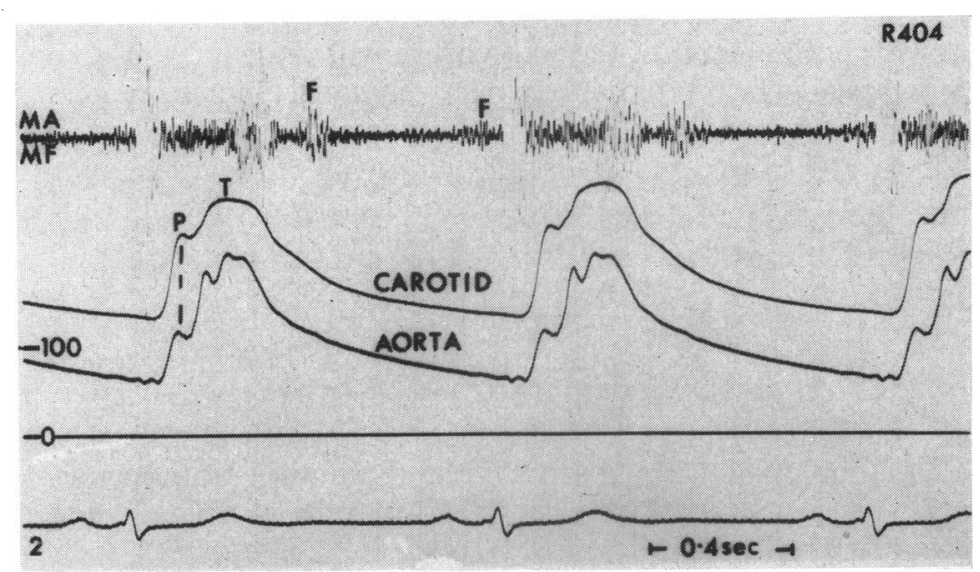

FIG. 8.-A case of aortic regurgitation under treatment for arterial hypertension. The simultaneously recorded carotid arterial displacement curve, the aortic pressure pulse, the mitral area phonocardiogram (MA/MF), and the electrocardiogram (2). $\quad F$ and $F=$ the Austin Flint murmur in early diastole and atrial systole.

\section{$63 \cdot 169$}

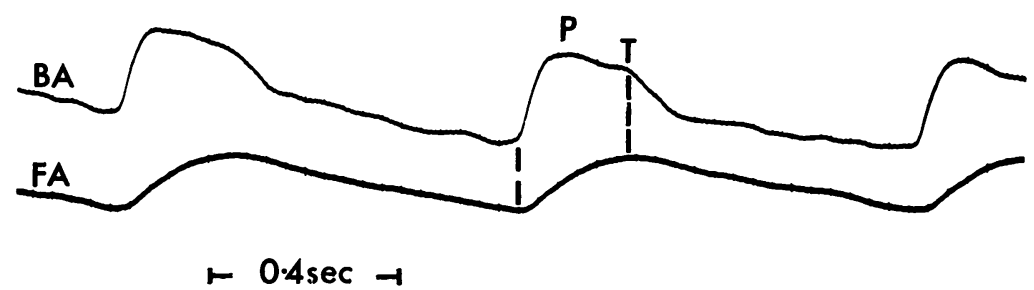

FIG. 9. - Coarctation of the aorta. Synchronously recorded brachial (BA) and femoral (FA) arterial displacement curves. The upstrokes begin at approximately the same time, but the percussion wave does not appear on the femoral curve.

the diastolic blood pressure, reduction in the stroke output, and aortic stenosis tend to oppose its production. There may be fewer difficulties if it is accepted that the factors unrelated to the aortic lesion may dominate the pulse. For example, in patients with aortic stenosis, the pulse may lose its anacrotic character when the heart accelerates (Bramwell, 1937). Another example is seen in a case of aortic regurgitation with arterial hypertension (Fig. 8). The Braunwald test showed that the volume of regurgitation was comparable with that of the patients with a bisferiens pulse who are reported in this study, and there was no evidence of stenosis, but the carotid tracing failed to show a bisferiens type of percussion wave, and there was little disproportion between the carotid displacement curve and the aortic pressure pulse. Presumably the condition of the arterial wall prevented the appearance of a large percussion wave.

\section{SUMMARY}

A bisferiens carotid arterial displacement pulse was recorded in 10 patients with severe aortic valvular disease. The Braunwald test indicated that moderately severe, severe, or gross aortic regurgitation was present in each. The absence of a peak systolic aortic pressure gradient in certain cases, particularly in those with the most severe regurgitation, suggested their freedom from an element of stenosis. 
The anacrotic wave and the peak of the aortic pressure pulse were found to coincide with the percussion and the tidal waves of the simultaneously recorded carotid arterial displacement curves. It is suggested that amplitude of the peak of the anacrotic wave is proportional to the rate of change of momentum of the ejected blood, and that the amplitude of the corresponding percussion wave of displacement is modified by the distensibility of the artery. The depth and shape of the trough between the two peaks is thought to depend upon the magnitude and slope of the two waves rather than upon a Venturi effect.

It is suggested that the condition of the arterial wall, the diastolic blood pressure, and the stroke volume can influence the pulse sufficiently to preclude its use as a simple guide to the nature of the aortic valve lesion.

The authors are grateful to Mr. G. H. Wooler for providing the facilities for this study; to Mr. R. Addyman and Mrs. M. Williams for assistance; and to the Board of Governors of the United Leeds Hospitals, the Nuffield Foundation, the Medical Research Council, and private benefactors for providing equipment. They wish to thank Dr. Arthur Hollman for providing a great deal of the historical information.

\section{REFERENCES}

Bramwell, C. (1937). The arterial pulse in health and disease. Lancet, 2, 239, 301, 366.

Braunwald, E., and Morrow, A. G. (1958). A method for the detection and estimation of aortic regurgitant flow in man. Circulation, 17, 505.

Broadbent, W. (1899). Pulsus bisferiens. Brit. med. J., 1, 75.

C, and Broadbent, J. F. H. (1900). Heart Disease, 3rd ed. Baillière, Tindall and Cox, London.

Clarke, J. M. (1894). On the pulsus bisferiens of aortic regurgitation. Lancet, $2,1529$.

Feil, H. S., and Gilder, M. D. D. (1921). The pulse in aortic disease as felt and graphically inscribed. Heart, 8, 4. Fleming, P. R. (1957). The mechanism of the pulsus bisferiens. Brit. Heart J., 19, 519.

Hancock, E. W., and Abelmann, W. H. (1957). A clinical study of the brachial arterial pulse form, with special reference to the diagnosis of aortic valvular disease. Circulation, 16, 572.

Hope, J. (1839). A Treatise on the Diseases of the Heart and Great Vessels, 3rd ed. Churchill, London.

Lewis, T. (1906). The pulse in aortic disease; the relation of pulse curves to blood pressure. Lancet, 2,714

McDonald, D. A., and Taylor, M. G. (1959). The hydrodynamics of the arterial circulation. Progr. Biophys., 9, 105.

Mackenzie, J. (1908). Diseases of the Heart. Oxford University Press, London.

Morison, A. (1894). On an avoidable error in sphygmography. Lancet, 2, 1531.

Nixon, P. G. F., Hepburn, F., and Ikram, H. (1964). Simultaneous recording of heart pulses and sounds. Brit. med.J., 1, 1169.

Robinson, B. (1963). The carotid pulse. II : Relation of external recordings to carotid, aortic, and brachial pulses. Brit. Heart J., $25,61$.

Ross, J., Jr. (1959). Catheterization of the left heart through the inter-atrial septum. Surg. Forum, 9, 297.

Schneider, H., and Klunhaar, E. W. J. M. (1961). Precordial low-frequency displacements of the thoracic wall. Method of recording and registration. Amer. Heart J., 61, 670.

Spencer, M. P., and Greiss, F. C. (1962). Dynamics of ventricular ejection. Circulat. Res., $10,274$.

Steell, G. (1894). The pulse of aortic stenosis. Lancet, 2, 1206.

Wood, P. (1956). Diseases of the Heart and Circulation, 2nd ed. Eyre and Spottiswoode, London. 